\title{
DNA-based switchable devices and materials
}

\section{Dongsheng Liu*, Enjun Cheng and Zhongqiang Yang \\ Tsinghua University, China}

Other than carrying the code of life, in the past three decades, DNA has played an important role in weaving two- and even threedimensional nanostructures by taking advantage of its programmable sequences and precise recognition properties. The highly specific base-pairing interactions of DNA molecules not only allow for the building of static DNA nanostructures, but also enable the construction of machine-like devices capable of responding to external stimuli, such as $\mathrm{pH}$, small molecules, light, electricity, and temperature, thus performing tasks otherwise beyond our reach. This review summarizes DNA-based switchable devices and materials, particularly where the responsiveness of DNA is caused by changes in secondary structures, and more importantly, where such stimuli-responsive processes are reversible. This review covers DNA-based devices and materials from smart surfaces and responsive nanopores/nanochannels to DNA hydrogels, which are expected to be designed with even more intelligence in the future.

$\mathrm{S}$ tudies on DNA molecules have long been focused on its biological functions, since the discovery of the molecular structure of DNA by Watson and Crick [1] in 1953. DNA is known to be composed of adenine (A), guanine $(\mathrm{G})$, cytosine $(\mathrm{C})$ and thymine (T), and the Watson-Crick interaction of A-T and C-G base-pairing leads to the formation of the double-helix structure when sequences are complementary; this base-pairing process is also referred to as hybridization. Single-stranded DNA (ssDNA) is considered flexible, whereas double-stranded DNA ( $\mathrm{d} s \mathrm{DNA}$ ) has a persistence length of about $50 \mathrm{~nm}$. In the early 1980s [2], scientists started reconsidering the chemical composition of DNA, examining details such as chain structure, adjustable persistence length at the nanoscale, base-pair formation and programmable sequence. DNA quickly gained fresh recognition beyond strict biological roles and was rapidly introduced into the field of nanoscience as a new type of building block [3-8]. For example, DNA was used to fabricate nanostructures [9-14] from two-dimensional DNA nanostructures to three-dimensional curved nanostructures, as complex as spheres, ellipsoids, and flasks utilizing DNA origami techniques. DNA was also designed for the construction of molecular devices or machines that can generate nanoscale movement [15-17]. Besides static nanostructures, DNA could be applied to self-assembled structures [18,19] or integrated within other functional systems [20], utilizing properties of DNA such as specific recognition, chain-exchange reactions, specific enzyme reactions and secondary structure transformation to enable precise control of motion at the molecular level $[19,21]$ or change properties at the macro scale [22]. Such responsive and switchable properties allow molecular machine-like devices to be built. Several aspects of this field have been reviewed by Seeman [23], Liu and Liu [4], Simmel and co-workers [7,24], Bath and Turberfield [25] and Willner and co-workers $[5,6]$ in the past several years. The present review focuses on DNA-based devices and smart materials that can respond to external stimuli, resulting in conformational changes to DNA structures at the nanoscale and detectable changes in properties such as volume, wettability at the macroscopic scale or transduction of force to move objects. This responsiveness is recoverable on removal of the external stimulus. DNA-based devices are introduced relating to smart surfaces and nanopores/nanochannels, while DNA-based smart materials are

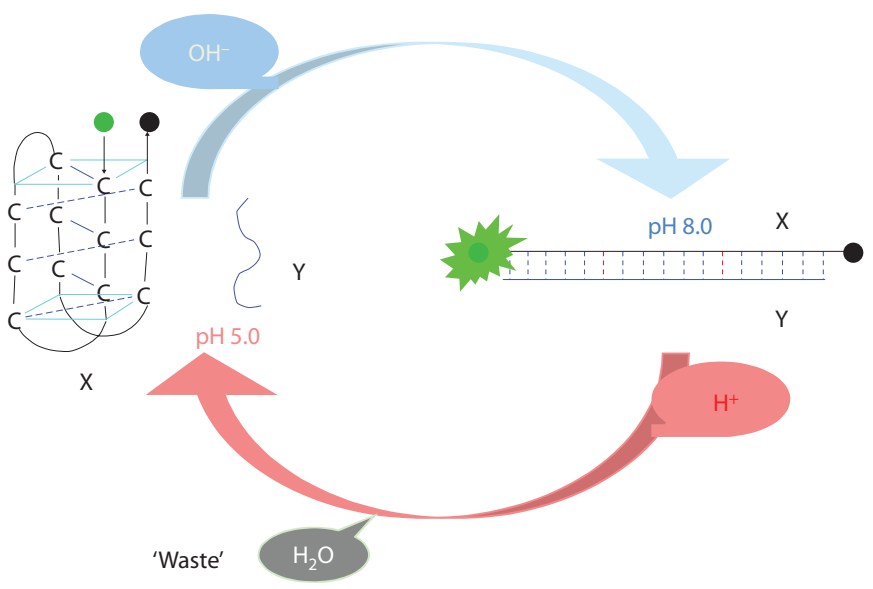

Figure 1. Schematic figure of the working cycle of i-motif DNA motors. At acidic pH, strand $X$ folds into the closed i-motif structure. When the $\mathrm{pH}$ is raised to 8.0, strand $\mathrm{X}$ unfolds and is captured by hybridization to $Y$ to form an extended duplex structure. Adapted from Ref. 19 (৫ 2003 Wiley-VCH).

related to newly developed pure and hybrid DNA hydrogels. Figure 1 shows a typical example of a DNA device or motor. In 2003, Liu and Balasubramanian [19] proposed a $\mathrm{pH}$-driven molecular motor system that is strong and swift. It comprises a 21 mer ssDNA sequence X containing four stretches of three cytosines and a 17 mer single-stranded DNA sequence $\mathrm{Y}$, which is partially complementary to $\mathrm{X}$. At $\mathrm{pH}$, via the formation of $\mathrm{C} \cdot \mathrm{CH}+$ base-pairs, sequence $\mathrm{X}$ folds into a compact 4-stranded i-motif structure with the 3' and 5' ends close to each other, representing the closed state of the motor. Changing the $\mathrm{pH}$ to 8 results in X unfolding and forming an extended DNA duplex structure XY, corresponding to the open state of the motor. These two states, compact and extended, can be reversibly switched by changing the $\mathrm{pH}$. Each stroke of the motor results in an output of a 5-nm linear movement that finishes in less than $1 \mathrm{~s}$. Moreover, the estimated opening/closing force outputs exceed $10 \mathrm{pN}$. The $\mathrm{i}$-motif structure has thus seen wide 


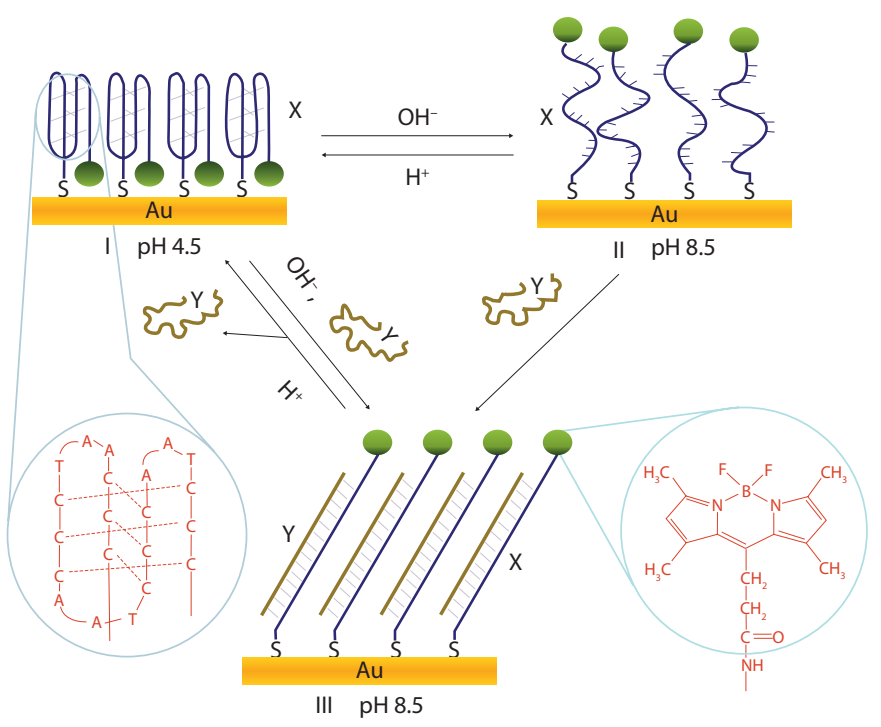

Figure 2. Reversibly wettability-switchable surface driven by DNA nanomotors. At low $\mathrm{pH}$, the DNA adopts an i-motif conformation (state I). Raising the $\mathrm{pH}$ destabilizes the i-motif to produce a stretched single-stranded state (state II) or duplex structure (state III, when a complementary strand is present). Lowering the $\mathrm{pH}$ induces a reverse conversion process from state II or III to state I. Adapted from Ref. 22 (c) 2007 Wiley-VCH).

use for constructing responsive DNA devices and materials. We hope this summary will benefit the application of smart devices and materials, allowing the development of new strategies for constructing new types of responsive systems and providing more precise and controllable DNA-based building blocks.

\section{Smart surfaces based on DNA}

A smart surface is a surface that can change its properties or enable certain functions in reaction to external stimuli. Its responsiveness normally results from the functional molecules modified onto the surface. Wellestablished methods of modifying DNA at the 3' and 5' ends have been widely used to make DNA-based functional surfaces like gene chips for sensing purpose [26,27]. However, 'smart' surfaces based on DNA have long been a challenge, due to the difficulties of achieving reversibility. In the case of adjusting surface properties, a densely packing (monolayer) of DNA and functional groups and synchronization of the movement of all components are necessary, requiring DNA structures sufficiently robust to undergo a predesigned performance. As we described previously, the DNA i-motif is a clean, quick, reliable and efficient molecular motor, exhibiting clear advantages over other examples [28] and providing many possibilities to facilitate smart surfaces based on DNA.

The proof-of-concept model to illustrate this DNA motor can still work at the solid-liquid interface has been developed by Liu et al. [21]. They introduced a 5'-thiol modification to the motor sequences, allowing a densely packed monolayer of DNA structures to be achieved on gold substrate via thiol-gold bonds. A fluorophore of rhodamine green was modified on the 3' end of a DNA motor as both cargo and reporting molecule. Typically, the metal surfaces can quench the fluorescence of a fluorophore, and the signal can be measured by fluorescence resonance energy transfer, so the distance between the fluorophore and surface is detectable and indicates the state of DNA strands. They investigated the assembly conditions and surface modifications and, using carefully designed control experiments, demonstrated that with the open status of the motor at high $\mathrm{pH}$, the fluorophore could be raised far from the gold surface with the formation of a duplex structure, and a bright fluorescent signal was observed under confocal microscopy. In contrast, at low $\mathrm{pH}$, the immobilized DNA strands transformed to the i-motif structure, bringing the fluorophore close to the gold surface. The fluorophore was thus quenched, and no obvious fluorescence could be observed. The change in distance with the motion of this DNA motor has also been verified by the monolayer height measured by atomic force microscopy in situ. Adjustment of $\mathrm{pH}$ values resulted in an effective and reversible transition between duplex and C-quadruplex, as confirmed by the observation of rapid switching of fluorescent signals. This demonstrated that the DNA motor based on i-motif structures is strong and suitably robust to operate at the interface and drive the motion of a small molecule, opening the door to the future development of smart surfaces.

Based on a similar strategy, Wang et al. [22] developed a three-state smart surface that can change wettability with changes in $\mathrm{pH}$. As illustrated in Figure 2, the motor DNA was assembled onto a gold surface through 5'-thiol modifications in a folded i-motif conformation at $\mathrm{pH} 5$ (state I), with the DNA phosphate-diester backbone, which was charged and hydrophilic, resulting in a hydrophilic surface. Under these conditions, a hydrophobic molecule (BODIPY 493/503 fluorophore) modified at the 3' ends of the DNA was hidden beneath the DNA monolayer. However, after changing $\mathrm{pH}$ to 8 (state II), DNA motors formed an elongated duplex structure, thus stretching out and exposing the hydrophobic BODIPY to solution, in the end changing surface wettability from hydrophilic to hydrophobic. The regulation of wettability was characterized by contact angle measurements. In that work, the authors also studied the influence of DNA chain effects and found a single-stranded state II was not very stable, mainly because the ssDNA was flexible and the hydrophobic BODIPY may become buried in the monolayer, exposing the hydrophilic DNA skeleton to solution. They also proved that by adding complementary strands of DNA motors to the system to form duplexes (state III), as the dsDNA was more rigid than ssDNA and the density of DNA strands increased, this not only made the DNA strands more packed, but also avoided spreading or rearrangement, eventually leading to a more stable and robust monolayer. Their studies also showed that this DNA modification technique has good adaptability and could be combined onto rough substrates, with the effect of motion from DNA motors potentially amplifying hydrophilicity or hydrophobicity to superhydrophilicity or superhydrophobicity, respectively. The study verifies the working ability of the DNA motor and shows its potential for doing much heavier work. In 2009, Meng et al. [29] further demonstrated that this molecular motor could be modified with quantum dots at 3' ends through covalent bonds, and as the i-motif DNA stretched or shrunk with $\mathrm{pH}$ changes, the quantum dots were pushed up and down, respectively, resulting in a controllable photoelectric conversion.

Besides moving functional molecules up and down, conformational changes in a DNA machine could be used in fabricating surfaces with controllable release properties. In 2004, Mao et al. [30] proposed a reversibly switchable DNA nanocompartment on surfaces. In 2007, Mao et al. [20] verified this proposition by conducting a series of experiments. They first started with a DNA monolayer possessing a folded four-stranded i-motif structure, which served as a nanocontainer and trapped small molecules (e.g. ferricyanide anions $\left.\left[\mathrm{Fe}(\mathrm{CN})_{6}\right]_{3}\right)$ inside. As the i-motif structure unfolded with adjustment of the $\mathrm{pH}$ environment from 4.5 to 8.0, the container was switched from open to closed, thus releasing molecules (Figure 3(a)). This $\mathrm{pH}$-triggered responsiveness greatly improves the efficiency of open-close process and their capacity, and provides more choice for fabricating controllable drug delivery systems.

Finally, the forces generated by DNA motors were measured using microcantilevers. In 2005, Shu et al. [31] demonstrated that numerous nanomachines in a monolayer achieved cooperative movement and changed the surface tension significantly, even drove a micro-cantilever bend. Cantilevers were coated with a thin layer of gold on one-side and then monolayer i-motif DNA motors in duplex form were assembled onto the surface (Figure $3(\mathrm{~b})$ ). Decreasing $\mathrm{pH}$ to 5 could lead to the transition of DNA motors from duplex form to i-motif form, and cause more than $100 \mathrm{~nm}$ of bending of the motor cantilever compared to control systems that did not achieve changes in the secondary structure. The magnitude of this surface stress difference has been estimated at about $32 \pm 3 \mathrm{mN} \mathrm{m}^{-1}$, which corresponds to a single motor force of 

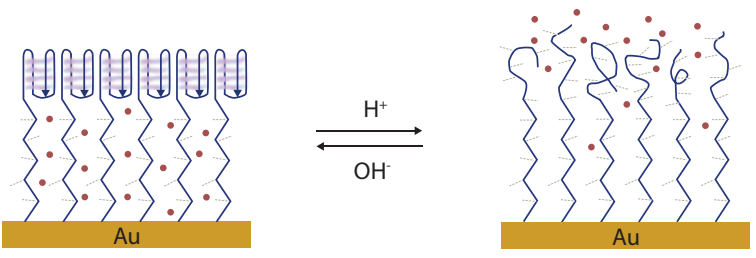

b

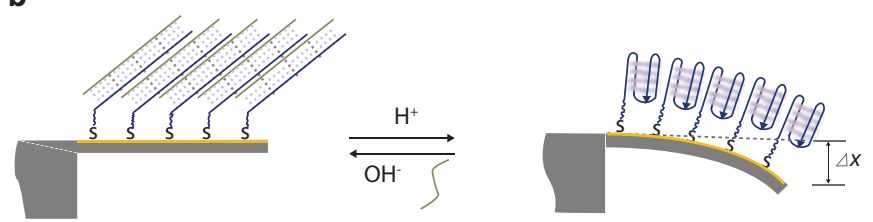

Figure 3. Illustration of (a) the bending process of the cantilever with an i-motif DNA motor and (b) the working principle for a switched DNA container. (a) At pH 4.5, the i-motif domain folds into a four-stranded structure and packs into a membrane impermeable to small molecules on the gold surfaces. At pH 8.0, the i-motif structures are transformed into single strands, making the packing density of the DNA self-assembled monolayer relatively loose and allowing small molecules to diffuse through freely. Adapted from Ref. 20 (๔ 2007 Mao et al.). (b) At pH 5.0, the DNA attached to the cantilever forms a self-folded i-motif and induces repulsive in-plane surface forces (compressive surface stress) which cause the cantilever to bend downward. Adapted from Ref. 31 (๔ 2005 ACS).

approximately $11 \mathrm{pN} \mathrm{m}^{-1}$. The surface stress originated mainly from electrostatic repulsion, as repulsion of the $\mathrm{i}$-motif structure is higher than that of the duplex form. The transition from duplex to four-stranded i-motif thus led to larger compressive stress and induced bending of the cantilever. Moreover, this bending process was highly reversible on $\mathrm{pH}$ change. That work was the first example to illustrate that the cooperative effects of DNA nanomotors could generate a dramatic force large enough to move macro objects [3]. The findings directly demonstrate the working ability of DNA molecular machines and opens the possibility of building smart mechanical devices at the nanoscale.

It is worth mentioning that operating a nanoscale molecular machine needs an accurate and precise system of input. The reported examples are mostly operated by adding chemicals or fuel DNA molecules that are easily realized in bulk systems, but not under nanoscale. In 2005, Leidl et al. [32] reported autonomous switching of DNA motor and surface optical signals for several times using an improved $\mathrm{pH}$ oscillator system. In 2007, Liu et al. [33] reported a light-driven method, enabling non-contact driving of the i-motif DNA motor for the first time. In 2010, Yang et al. [34] achieved autonomous control of the DNA motor using electrical signals. That method is very reliable, fast and programmable, offering an ideal complement to pH-driven DNA molecular motors. This technical advance will also benefit the application of DNA molecular motors in constructing complicated surface-based nanodevices.

\section{DNA responsive nanopore/nanochannel}

Nanopores and nanochannels have attracted much attention for a long time because of their importance in biological activities. To understand the transportation mechanism and behavior of ions, biomolecules in the pore/channel and polymer and inorganic nanopores/nanochannels have been created. To confer on these nanopores/nanochannels stimuliresponsive properties to mimic their natural models, polymers $[35,36]$, peptides [37] and DNA [38-40] have been modified onto the inner surface of nanopores/nanochannels. Among these studies, DNA has been demonstrated as an outstanding molecule to create an intelligent nanopore/nanochannel because of the clear transformation of secondary structure.

In 2004, by attaching hairpin DNA to the inner surface of nanochannels, Kohli et al. [41] made a smart nanochannel that could selectively
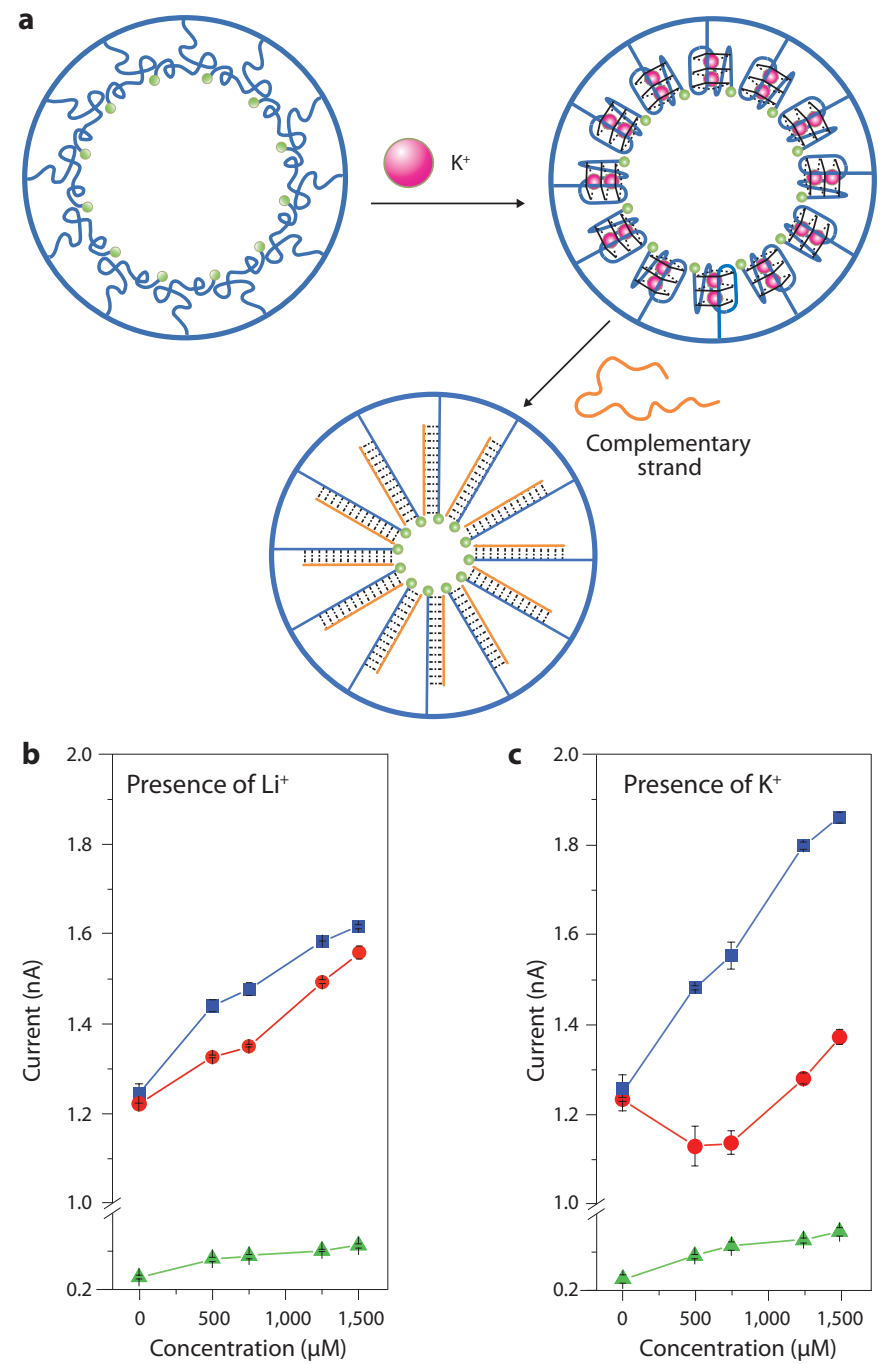

Figure 4. Schematic figure of (a) a potassium (shown as pink) sensitive nanopore/ nanochannel, $(b, c)$ current-concentration $\mathrm{Li}^{+}(\mathrm{b})$ and $\mathrm{K}^{+}(\mathrm{c})$ properties of the single nanopore/nanochannel before and after DNA molecules (shown as blue) attached to the inner wall in Tris- $\mathrm{HCl}\left(5 \mathrm{mM}, \mathrm{pH} 7.2\right.$, at $\left.23^{\circ} \mathrm{C}\right)$. G-quadruplex DNA was immobilized onto a synthetic nanopore/nanochannel, which undergoes a potassium-responsive conformational change and then induces the change in effective pore size. Adapted from Ref. 39 (๔ 2009 ACS).

recognize and transport the DNA strand that is complementary to the hairpin strand. In 2008, Xia et al. [39] covalently attached i-motif DNA into an asymmetric poly(ethylene terephthalate) nanochannel and achieved a pH-gating nanopore/nanochannel; in other words, the nanopore/nanochannel is able to regulate ionic transport properties by tuning $\mathrm{pH}$. The nanopore/nanochannel has a $\mathrm{pH}$-responsive conductivity change and the authors proposed that the mechanism was related to the charge densities of different DNA conformations. One year later, Hou et al. [40] further studied the response mechanisms of DNAmodified nanopore/nanochannel with a potassium ion responding to a DNA sequence that is guanine $(G)$ rich, and can fold into a four-stranded G-quadruplex in the presence of potassium ions. In the presence of lithium, they demonstrated that the G-rich sequences adopted a loosely packed single-stranded structure inside the channel (Figure 4). This conformation is believed to be very flexible in a random-coiled state and has no obvious influence on the transportation of ions through the channel, and observation of the current/ion concentration confirmed a monotonous linear relation. This behavior was the same as that in a bare channel without DNA modification and further verified that randomly coiled ssDNA will not influence the transportation of ions. In the presence of 

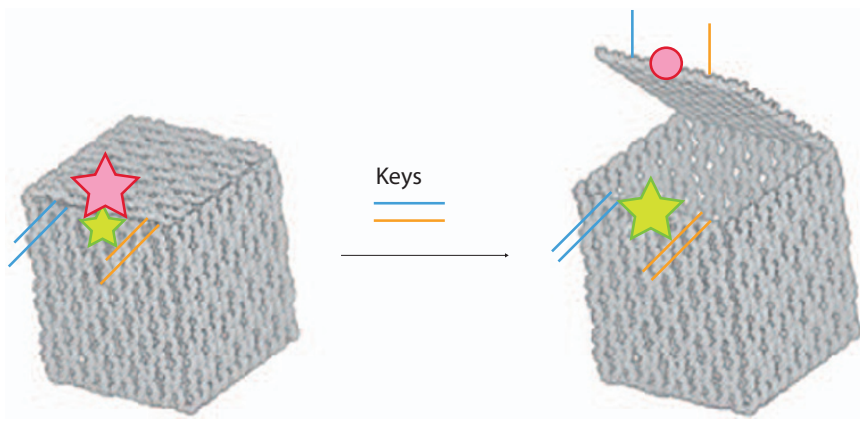

Figure 5. Illustration of the controlled opening process of the DNA box. The DNA box with a DNA 'smart' locker can be opened and closed by an externally supplied DNA 'key'. Emissions from the Cy3 and Cy5 fluorophores are marked with green and red stars, respectively. Loss of emission from Cy 5 is denoted by a red circle and the independent lock-key systems are indicated in blue and orange. Adapted from Ref. 11 (๔ 2009 NPG).

potassium, the G-rich DNA strands will adopt a four-stranded conformation, with highly packed chain and a high density of negative charges near the channel wall, which is not permeable to ions, and subsequently decreased the effective size at the narrowest point of the channel, thus decreasing the current. Of these two factors, ion concentration has a positive effect (more ions pass) and the presence of G-quadruplex exerts a negative effect (the compact structure blocks ion transfer through the nanopore/nanochannel). Taken together, this results in interesting behaviors for this nanopore/nanochannel (Figure 4(b,c)): current first starts to drop with an increase in the concentration of potassium ions from 0 to $500 \mu \mathrm{M}$, which could be attributed to the increasing proportion of newly formed G-quadruplex; and with concentrations above $500 \mu \mathrm{M}$, currents return to a monotonous linear relation with potassium ion concentration. Hou et al. [40] proposed that this was because all DNA strands were folded into the G-quadruplex under this condition. This result and proposed mechanism shows good agreement with a previous nanocontainer study. They also showed that when fully matched complementary sequences were added to hybridize with the DNA modified onto the channel wall to form a stable duplex, although lithium or potassium ions were present, the channel only behaved like a bare channel of smaller size. This verified that the high density of the DNA chain can obscure the transport of ions in the nanopore/nanochannel. A better understanding of the effects of DNA structures in smart nanopore/nanochannels will allow the design and fabrication of intelligent nanopore/nanochannels with precise and controllable mechanisms.

\section{Other DNA-responsive nanodevices}

The development of DNA-based responsive devices has been quick and diverse. There are many interesting examples that cannot be simply attributed to the above-mentioned smart surfaces or responsive nanopore/ nanochannels, but which are also very important and might inspire future direction in this field. For example, Wang et al. [42] prepared an artificial DNA nanospring formed from circular DNA connected by a DNA i-motif structure. At low $\mathrm{pH}$, this nanospring was in a compressed state, while changing $\mathrm{pH}$ to 8 hybridized the $\mathrm{i}$-motif structure with a full complementary sequence forming dsDNA, inducing stretching of the nanospring. Recently, the same group also used i-motif DNA coupled to mesoporous silica nanoparticles to demonstrate a novel proton-fueled molecular gate-like delivery system for the controllable release of small molecules [43]. In 2008, the 'smart' design was also introduced into the DNA origami technique, with Andersen et al. [44] reporting a DNA dolphin that was flexible and could swing its tail by adjusting both force and scanning direction of the atomic force microscopy tip. Soon thereafter, the same group prepared a three-dimensional DNA box with a DNA 'smart' locker, which can be open and closed using a special DNA a
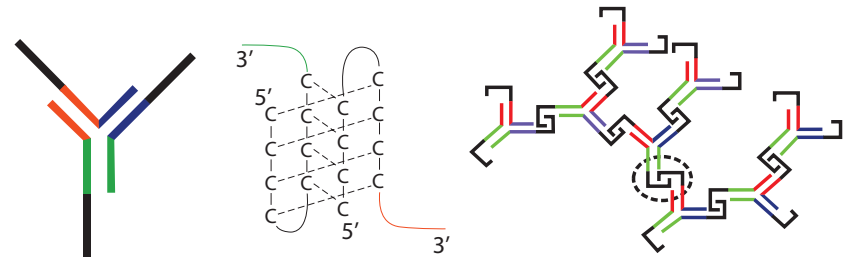

b
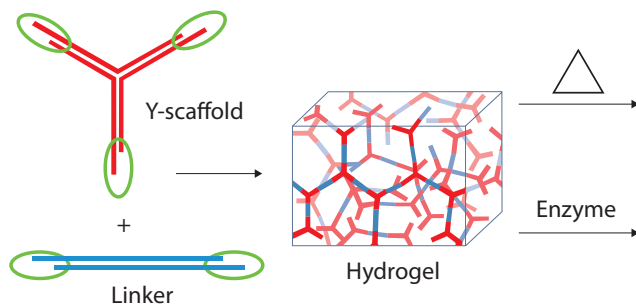

Hydrogel

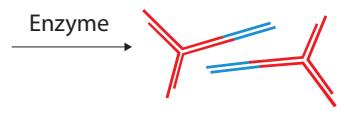

Figure 6. Pure DNA hydrogels with (a) pH responsiveness and (b) heat and enzymatic dual responsiveness. (a) A Y-shaped DNA nanostructure is formed from three singlestranded DNAs with two functional domains: an interlocking i-motif domain containing two cytosine-rich stretches (marked in black); and a domain for formation of the double-stranded $Y$ shape (marked in red, green, and blue). The inter-Y-unit i-motif structures can be formed and leads to an extended three-dimensional network in the DNA hydrogel. Adapted from Ref. 54 (@ 2009 Wiley-VCH). (b) The Y-scaffold and Linker are designed to crosslink by hybridization of the 'sticky ends' (emphasized by green circles). By tailoring these 'sticky ends' and inserting restriction sites in the Linker sequences, the DNA hydrogels show thermal and enzymatic responsive properties. Adapted from Ref. 55 (๔ 2010 Wiley-VCH).

'key' [11] (Figure 5). To detect the opening process, they functionalized the box with $\mathrm{Cy} 3$ (donor) and Cy5 (acceptor) fluorophores and a lock-key system. The opening of the box was directly monitored in fluorescence kinetic experiments, during which DNA 'keys' were added to the closedbox. In 2010, Chen et al. [45] showed that the pH-driven DNA molecular motor can even drive two synthetic dendrons to associate and dissociate. These results illustrate not only the elegance of DNA nanotechnology, but also provide more imagination space for future applications. Mention is also needed of the fact that the ability to move molecules, such as fluorescent dyes, using DNA motors has been widely used in building sensors, which have been widely reported and recently reviewed [46-51].

\section{Pure DNA hydrogel}

DNA molecules have now been well acknowledged as building blocks for fabricating smart devices and precisely addressable structures at the nanometer scale, far from the common understanding of 'bulk' materials. In 2006, by sacrificing some accuracy such as precise structures and conformations, Um et al. [52] prepared 'bulk' structures with a simple strategy. They designed several DNA short chains, which can assemble into multi-branched DNA structures. These structures possess selfcomplimentary sticky ends, and can further assemble via subsequent enzyme ligation between each other to form a network, termed a 'DNA hydrogel'. These gels could be loaded with insulin, which was released over time on degradation of the DNA. By employing less complex DNA structures consisting of fewer DNA chains and scaling up production, the cost of this hydrogel on average could be decreased to an acceptable level. Later, the same group also successfully incorporated a functional plasmid into the hydrogel and invented a cell-free protein-producing hydrogel system [53].

In 2009, Cheng et al. [54] reported a pH-responsive pure DNA hydrogel whose formation could avoid utilizing enzymes, which is a potential immune response factor. In their work, three ssDNAs assembled into a Y-shaped ternary complex with interlocking domains at each terminus that can form intramolecular i-motif structures (Figure 6(a)). At neutral $\mathrm{pH}$, no interactions existed within the assembled Y-shaped 
DNA structure because of electrostatic repulsion. By decreasing the $\mathrm{pH}$ to 5 , the i-motif domain became half-protonated and then formed intermolecular i-motif complexes between adjacent Y-shaped structures, resulting in a hydrogel. The transition from solution state to gel state could occur within 1 minute. The formed gel is much stronger than previously reported samples, potentially attributable to the smaller spatial hindrance of the proton than the ligation reagent: protons were smaller than enzymes and could disperse more easily within the partially crosslinked network. This not only increased the assembly efficiency, but also led to a higher crosslinking density. It is also worth noting that $13 \mathrm{~nm}$ gold nanoparticles, as a model molecule, could be trapped and released from the hydrogel matrix following $\mathrm{pH}$ changes, demonstrating potential as a smart drug carrier.

Xing et al. [55] recently reported a new strategy to prepare pure DNA hydrogels. They designed two kinds of building blocks, in the form of Y-shaped and Linker DNA assemblies with complementary sticky ends (Figure 6(b)). Simply mixing the two building blocks at a proper concentration would induce the assembly of a Y-scaffold and Linker alternately by hybridization of the sticky ends. Accumulating this assembly process will lead to an expanded network, a DNA hydrogel. Changing the length and composition of sticky ends will confer different thermal stabilities on the system. In that report, they also designed a restriction enzyme sequence domain in the Linker domain, bringing specific enzymatic responsiveness to the hydrogel besides the adjustable thermal responsiveness. This research further widened the application conditions of DNA hydrogels.

\section{Hybrid DNA hydrogel}

Just a small portion of DNA is needed to achieve hydrogel responsiveness, in which the DNA normally acts as a responsive component and is present as a crosslinker [56-70]. In 1996, Nagahara and Matsuda [56] reported a DNA-polymer hybrid hydrogel. They grafted ssDNA chains onto polyacrylamide as side chains via chemical bonds, then mixed two kinds of this DNA side chain polymer possessing complementary ssDNA. Based on the formation of the duplex, a polymer/DNA hybrid hydrogel was able to be obtained. In 2004, Lin et al. [57] demonstrated that a hybrid hydrogel prepared with this strategy could transit from gel state to solid state on addition of a removal strand. Liedl et al. [59] subsequently employed this type of reversible DNA-polymer hybrid hydrogel to realize a controllable trap and release of quantum dots. We can also imagine that such a hybrid hydrogel could respond to thermal signals through deformation of the duplex. Theoretically, this strategy could allow hybrid hydrogel to be formed on all established DNA interlocking structures as well as duplexes. Wei et al. [61] prepared a DNA/polyacrylamide hydrogel in which the DNA crosslinking unit was a thrombinbinding aptamer (Figure 7). The strategy also brings to the hydrogel a versatile responding mechanism. For example, Yang et al. [62] reported an aptamer-based sensing device in which the presence of adenosine triggered dissolution of a hydrogel. Their subsequent work used the same strategy to design a visual detection method for sensing cocaine. The versatility of this approach might be adaptable to the visual detection of a wide variety of molecules that could benefit biosensing and medical applications $[63,66]$.

As mentioned previously, ssDNA is very flexible and dsDNA possesses a much bigger persistence length of about $50 \mathrm{~nm}$, so ssDNA and dsDNA can induce significant changes in persistence length based on conformational changes. As a result, a large volume change can be achieved on stimulation, and can be recovered with removal of the stimulus. In 2005, Murakami and Maeda demonstrated that, by chemically coupling ssDNA into a crosslinked polyacrylamide hydrogel, the addition of particular oligonucleotide sequences could trigger the shrinkage or expansion of the hybrid hydrogel, specifically in the form of sequence-dependent volume transitions [68,69]. Tierney and Stokke [70] also reported an interferometric readout platform capable of determining changes in the optical length of a DNA hybrid hydrogel.

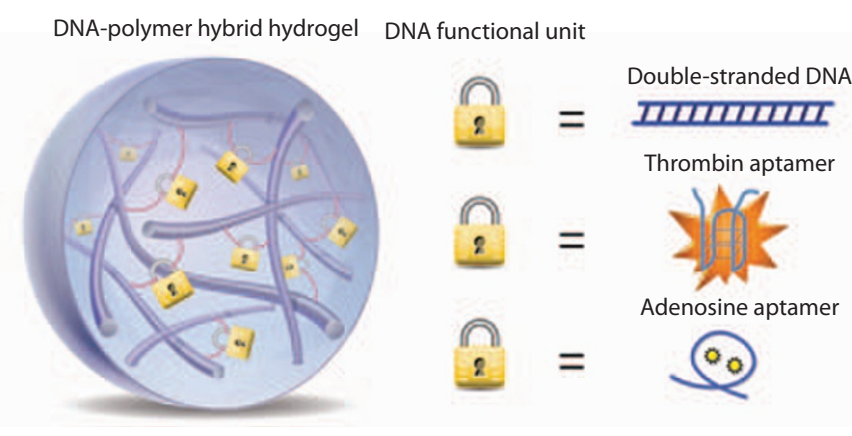

Figure 7. Schematic diagram of a DNA-polymer hybrid hydrogel, a DNA functional unit working as a crosslinker, which can be referred to as a double-stranded DNA [56], a thrombin aptamer [61] or adenosine aptamer [62]. This DNA-polymer strategy could provide the hydrogel with a versatile responding mechanism.

An optical-fiber apparatus was incorporated with partially ssDNAlinked polyacrylamide gel, which could be swollen by introducing a target sequence to competitively hybridize with DNA grafted into the polyacrylamide chain. This apparatus was capable of resolving changes in swelling height with 2-nm resolution, depending on the optical path length through the gel. This sensing device was label-free and sensitive to both DNA sequence and concentration, which could provide a versatile sensing platform for a variety of biomolecular targets.

This field could be further expanded by varying the components. For example, Cheng et al. [71] wrapped ssDNA onto a single-wall carbon nanotube via charge transfer from the bases of DNA to the carbon nanotubes. Working as a crosslinker unit, ssDNA further linked with extensive long assembled DNA chains to form three-dimensional structures, creating a $\mathrm{pH}$-responding, strength-tunable hybrid hydrogel. In 2010, Xu et al. [72] prepared a DNA-graphene hybrid hydrogel using similar interactions as in the case of wrapping carbon nanotubes with DNA. The hybrid hydrogel is capable of self-healing with heat, since the free dsDNA opens the double helix structure and forms ssDNA on heating, which in situ binds to graphene sheets and repairs the damage.

\section{Conclusion and perspective}

The ability to undergo environment-stimulated conformational transitions has made DNA a popular stimulus-responsive element for creating smart devices and materials, and the response mechanism has also expanded from well-known chain exchange reactions and enzyme restriction reactions to $\mathrm{pH}$ changes, small molecules and even light and electrical signals. We believe that the excellent adaptability of DNA, with inspiration from all the reported strategies and further developments in material science and nanotechnology, will enable even more exciting smart systems in the future and will greatly benefit the invention of portable medical devices. Owing to its biocompatibility, DNA-based smart materials, particularly pure DNA hydrogels, will play increasingly important roles in implantable materials and controllable drug/gene delivery systems. In the meantime, many challenges remain in this emerging field, such as how the reliability of DNA smart devices and materials can be improved, and how DNA smart devices can be made to more efficiently convert energy into work. New tools and strategies are also needed to measure the output force of DNA machine directly at the single molecule level. These studies will benefit not only chemistry and materials science, but also lead to advances in physics, biology and medical research.

\section{Acknowledgments}

The authors received funding support for their research from the National Basic Research Program of China (973 program, Nos 2007CB935900 and 2011CB935701) and the National Natural Science Foundation of China (Nos 20725309 and 91027046). 


\section{References}

1. J. Watson, F. C. Crick, Nature 171, 737 (1953).

2. N. C. Seeman, J. Theor. Biol. 99, 237 (1982).

3. T.Liedl, T. L. Sobey, F. C. Simmel, Nano Today 2, 36 (2007).

4. H. Liu, D. Liu, Chem. Commun., 2625 (2009).

5. C. Teller, I. Willner, Curr. Opin. Biotech.. 21, 376 (2010)

I. Willner, B. Willner, Nano Lett. 10, 3805 (2010).

Y. Krishnan, F. C. Simmel, Angew. Chem. Int. Ed. 50, 3124 (2011).

L. J. Xu, D. S. Liu, Chem. Soc. Rev. 39, 150 (2010).

P. W. K. Rothemund, Nature 440, 297 (2006).

Y. He et al., Nature 452, 198 (2008).

E. S. Andersen, et al., Nature 459, 73 (2009).

H. Dietz, S. M. Douglas, W. M. Shih, Science 325, 725 (2009).

S. M. Douglas et al., Nature 459, 414 (2009).

D. Han et al., Science 332, 342 (2011).

15. W. B. Sherman, N. C. Seeman, Nano Lett. 4, 1203 (2004).

16. P. Yin, H. Yan, X. G. Daniell, A. J. Turberfield, J. H. Reif, Angew. Chem. Int. Ed. 43, 4906 (2004)

17. K. Lund et al., Nature 465, 206 (2010).

18. J. W. J. Li, W. H. Tan, Nano Lett. 2, 315 (2002).

19. D. S. Liu, S. Balasubramanian, Angew. Chem. Int. Ed., 42, 5734 (2003).

20. Y. D. Mao et al., Nucleic Acids Res. 35, e33 (2007).

21. D. S. Liu et al., J. Am. Chem. Soc. 128, 2067 (2006).

22. S. T. Wang et al., Angew. Chem. Int. Ed. 46, 3915 (2007).

23. N. C. Seeman, Trends Biochem. Sci. 30, 119 (2005).

24. F. C. Simmel, W. U. Dittmer, Small 1, 284 (2005).

25. J. Bath, A. J. Turberfield, Nature Nanotechnol. 2, 275 (2007).

26. C. Fan, K. W. Plaxco, A. J. Heeger, P. Natl. Acad. Sci. USA 100, 9134 (2003).

27. S. J. Park, T. A. Taton, C. A. Mirkin, Science 295, 1503 (2002).

28. B. Yurke, A. J. Turberfield, A. P. Mills, F. C. Simmel, J. L. Neumann, Nature 406, 605 (2000).

29. H. Meng et al., Chem. Commun., 2293 (2009).

30. Y. Mao et al., Nucleic Acids Research 32, e144 (2004).

31. W. M. Shu et al., J. Am. Chem. Soc. 127, 17054 (2005).

32. T. Liedl, M. Olapinski, F. C. Simmel, Angew. Chem. Int. Ed. 45, 5007 (2006).

33. H. J. Liu et al., Angew. Chem. Int. Ed. 46, 2515 (2007).

34. Y. Yang et al., Nano Lett. 10, 1393 (2010).

35. X. Hou, L. Jiang, ACS Nano 3, 3339 (2009).

36. X. Hou, W. Guo, L. Jiang, Chem. Soc. Rev. 40, 2385 (2011).

37. Y. Jung, H. Bayley, L. Movileanu, J. Am. Chem. Soc. 128, 15332 (2006).
38. R. Fan et al., Nano Lett. 5, 1633 (2005).

39. F. Xia et al., J. Am. Chem. Soc. 130, 8345 (2008).

40. X. Hou et al., J. Am. Chem. Soc. 131, 7800 (2009).

41. P. Kohli et al., Science 305, 984 (2004)

42. C. Y. Wang, Z. Z. Huang, Y. H. Lin, J. S. Ren, X. G. Qu, Adv. Mater. 22, 2792 (2010).

43. C. Chen et al., Nucleic Acids Research 39, 1638 (2011).

44. E. S. Andersen et al., ACS Nano 2, 1213 (2008).

45. P. Chen et al., Soft Matter 6, 2143 (2010).

46. D. Li, S. P. Song, C. H. Fan, Acc. Chem. Res. 43, 631 (2010).

47. X. Duan, L. Liu, F. Feng, S. Wang, Accounts Chem. Res. 43, 260 (2009).

48. B. Juskowiak, Anal. Bioanal. Chem. 399, 3157 (2011).

49. I. Willner, M. Zayats, Angew. Chem. Int. Ed. 46, 6408 (2007).

50. J. Liu, Z. Cao, Y. Lu, Chem. Rev. 109, 1948 (2009).

51. E. J. Cho, J. W. Lee, A. D. Ellington, Annu. Rev. Anal. Chem. 2, 241 (2009).

52. S. H. Um et al., Nat Mater. 5, 797 (2006).

53. N. Park, S. H. Um, H. Funabashi, J. F. Xu, D. Luo, Nature Mater. 8, 432 (2009).

54. E. J. Cheng et al., Angew. Chem. Int. Ed. 48, 7660 (2009).

55. Y. Xing et al., Adv. Mater. 23, 1117 (2011).

56. S. Nagahara, T. Matsuda, Polym. Gels Netw. 4, 111 (1996).

57. D. C. Lin, B. Yurke, N. A. Langrana, J. Biomech. Eng.-Trans. ASME 126, 104 (2004).

58. D. C. Lin, B. Yurke, N. A. Langrana, J. Mater. Res. 20, 1456 (2005).

59. T. Liedl, H. Dietz, B. Yurke, F. C. Simmel, Small 3, 1688 (2007).

60. F. X. Jiang, B. Yurke, B. L. Firestein, N. A. Langrana, Ann. Biomed. Eng. 36, 1565 (2008).

61. B. Wei, I. Cheng, K. Q. Luo, Y. L. Mi, Angew. Chem. Int. Ed. 47, 331 (2008).

62. H. H. Yang, H. P., Liu, H. Z. Kang, W. H. Tan, J. Am. Chem. Soc. 130, 6320 (2008).

63. X. J. He, B. Weiz, B., Y. L. Mi, Chem. Commun. 46, 6308 (2010)

64. F. X. Jiang, B. Yurke, R. S. Schloss, B. L. Firestein, N. A. Langrana, Tissue Eng. Part A 16, 1873 (2010).

65. F. X. Jiang, B. Yurke, R. S. Schloss, B. L. Firestein, N. A. Langrana, Biomaterials 31, 1199 (2010).

66. Z. Zhu et al., Angew. Chem. Int. Ed. 49, 1052 (2010).

67. H. Z. Kang et al., Langmuir 27, 399 (2011).

68. Y. Murakami, M. Maeda, Macromolecules 38, 1535 (2005).

69. Y. Murakami, M. Maeda, Biomacromolecules 6, 2927 (2005).

70. S. Tierney, B. T. Stokke, Biomacromolecules 10, 1619 (2009).

71. E. Cheng, Y. Li, Z. Yang, Z. Deng, D. Liu, Chem. Commun. 47, 5545 (2011).

72. Y. X. Xu, Q. O. Wu, Y. Q. Sun, H. Bai, G. Q. Shi, ACS Nano 4, 7358 (2010).

\section{Author profiles}

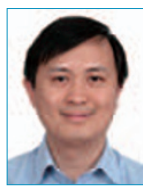

\section{Dongsheng Liu}

Dongsheng Liu graduated from the University of Science and Technology of China with a BS degree in 1993. After working in the Institute of Chemistry at the Chinese Academy of Sciences for six years, he attended Hong Kong Polytechnic University and finished his PhD there in 2002. He then worked as a postdoctoral research associate in NanolRC and the Department of Chemistry at Cambridge University, UK. In 2005, he bcame a principal investigator at the National Centre for NanoScience and Technology, China. From June 2009, he has been a full professor of the Department of Chemistry at Tsinghua University. His current research is mainly focused on using biomolecules to fabricate nanostructures and nanodevices.

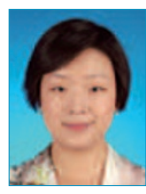

\section{Zhongqiang Yang}

Zhongqiang Yang received her BEngr (2000) and MSc (2003) degrees from Heilongjiang University and Jilin University in China, respectively. In 2003, she obtained a Gates Cambridge Trust and Overseas Research Studentship and pursued her PhD at the University of Cambridge, USA, at the Melville Laboratory for Polymer Synthesis. In 2007, she attended the University of Wisconsin-Madison, USA, carrying out postdoctoral work. In 2010, she joined the faculty of the Chemistry Department at Tsinghua University as associate professor. Her research interests include biomaterials, miniemulsions, liquid crystals and sensors.

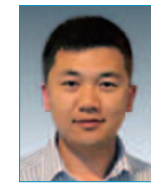

\section{Enjun Cheng}

Enjun Cheng received his BS degree in chemistry from Jilin University, China, in 2006. He then went to the National Center for Nanoscience and Technology, China, to pursue his doctoral degree from 2006 to 2011. During his PhD period, he worked on developing new biomaterials based on DNA molecules, including DNA hydrogels, DNA-carbon nanotube hybrid hydrogels and DNA-virus coat protein assembly structures. 